\title{
Vernetzt, flexibel und gesund?
}

\section{Status quo und Perspektiven der Regulierung von orts- und zeitflexibler Arbeit}

\author{
Mario Daum und Claus Zanker
}

\section{Zusammenfassung}

Der Wandel der Erwerbsarbeit infolge der Digitalisierung eröffnet neue Optionen aber birgt auch einige Gefahren. Die Autoren verfolgen in diesem Beitrag einen interdisziplinären Zugang zu orts- und zeitflexiblen Arbeitsformen, die durch den digitalen Wandel verstärkt möglich sind. Sie stellen die gegenwärtigen Forderungen nach Deregulierung bzw. Regulierung des Arbeitsrechts dar und ordnen diese auf Grundlage von Erkenntnissen der Arbeits- und Rechtswissenschaft ein. Auf ihren Ergebnissen basierend, geben sie Handlungsempfehlungen für Akteure aus Politik und Wirtschaft zur Gestaltung und Regulierung von orts- und zeitflexiblem Arbeiten.

\subsection{Einleitung}

Die Erwerbsarbeit in einer digitalisierten Welt ist mit einer Reihe von Spannungsfeldern verbunden, die sich durch die Digitalisierung von Arbeitsprozessen neu ergeben oder in ihrer Wirkung von diesen verstärkt werden. Eines dieser Spannungsfelder ist das zwischen Flexibilisierung und Arbeitsschutz, welches durch die digitale Transformation eine neue Qualität erfährt und vor neuen und wachsenden Herausforderungen steht. Durch die Nutzung digital-mobiler Arbeitsmittel und die Digitalisierung von Informationen als wesentliche Arbeitsgegenstände bei vielen insbesondere wissensbasierten Tätigkeiten ergeben sich erweiterte Möglichkeiten von örtlicher Mobilität und zeitlicher Flexibilität bei der Arbeit. Dies eröffnet einerseits Optionen für selbstbestimmtes Arbeiten und kann die

M. Daum $(\bowtie) \cdot$ C. Zanker

Projekt TransWork, INPUT Consulting gGmbH, Stuttgart, Deutschland

E-Mail: daum@input-consulting.de; zanker@input-consulting.de 
Vereinbarkeit von Beruf und Privatleben verbessern. Andererseits birgt es die Gefahr der Verwischung bisheriger Grenzen von Beruflichem und Privatem, was wiederum mit wachsenden Belastungen einhergehen kann. Eine Reihe von wissenschaftlichen Untersuchungen haben die Folgen der Digitalisierung auf die Arbeitswelt in den zurückliegenden Jahren in den verschiedenen Facetten analysiert. Gleichzeitig ist eine wissenschaftliche und politische Debatte darüber entbrannt, ob und wie sich die Regulierung von Arbeit angesichts dieses Wandels in der Arbeitswelt verändern muss.

Der Fokus dieses Beitrags liegt auf der Einordnung der arbeitsbezogenen Orts- und Zeitflexibilität sowie der interdisziplinären Analyse des Spannungsfeldes. Wir erachten die Zusammenführung der politisch geprägten Debatte hinsichtlich des arbeitsrechtlichen Handlungsbedarfs einerseits mit den wissenschaftlichen Erkenntnissen aus den Forschungsdisziplinen der Arbeitssoziologie, Arbeitswissenschaft (inkl. Arbeitsmedizin und Arbeitspsychologie) und des Arbeitsrechts andererseits als einen wertvollen Beitrag, um die wesentlichen Regelungslücken zu identifizieren. Dabei wird deutlich, dass manche Forderungen nach Veränderung der Arbeitsregulierung - wenig überraschend - stark interessengeleitet sind. Anhand unserer Analysen haben wir einige Maßnahmen für die Regulierung und Gestaltung abgeleitet, die den Ausgleich zwischen einem Mehr an Flexibilität und Selbstbestimmung einerseits sowie der Vermeidung von Selbstüberforderung und der Gewährleistung eines effektiven Arbeits- und Gesundheitsschutzes andererseits zum Ziel haben.

Der vorliegende Beitrag ist eine Kurzfassung unseres im Februar 2020 erschienenen TransWork-Projektberichts, für den wir die politische und wissenschaftliche Debatte und Literatur bis Oktober 2019 berücksichtigt haben.

\subsection{Digitale Arbeitswelt: Arbeit Zwischen Flexibilität und Entgrenzung}

Digitalisierte Arbeit ist Erwerbsarbeit, welche unter maßgeblicher Nutzung von informations- und kommunikationstechnologischen Arbeitsmitteln wie Computern, Tablets, Smartphones und sonstigen digitalen Geräten an Arbeitsgegenständen verrichtet wird, die in wesentlichen Anteilen als Informationen in digitalisierter Form existieren (vgl. Schwemmle und Wedde 2012, S. 14). Mit der zunehmenden Nutzung digitaler Arbeitsmittel und der wachsenden Bedeutung digitalisierter Informationsverarbeitung in vielen Berufen steigt der Anteil an Tätigkeiten, die unter dem Begriff der digitalisierten Arbeit zu subsumieren sind. Einer im Auftrag des Bundesministeriums für Arbeit und Soziales im Jahr 2015 durchgeführten Studie zufolge nutzen im Durchschnitt 83 Prozent der Beschäftigten bei ihrer beruflichen Tätigkeit digitale Technologien (Bundesministeriums für Arbeit und Soziales (BMAS) 2016a, b). Dabei zeigen sich jedoch in Abhängigkeit von Qualifikation und Berufsgruppe unterschiedliche Nutzungsraten digitaler Arbeitsmittel (siehe Abbildungen) (Abb. 2.1 und 2.2). 
Nutzen Sie bei Ihrer Tätigkeit digitale Informations- und Kommunikationstechnologien, wie z. B. Computer, Internet, Laptop, Tablet oder Smartphone? (Antworten "ja" in Betrieben mit mehr als 50 sozialversicherungspflichtig Beschäftigten)

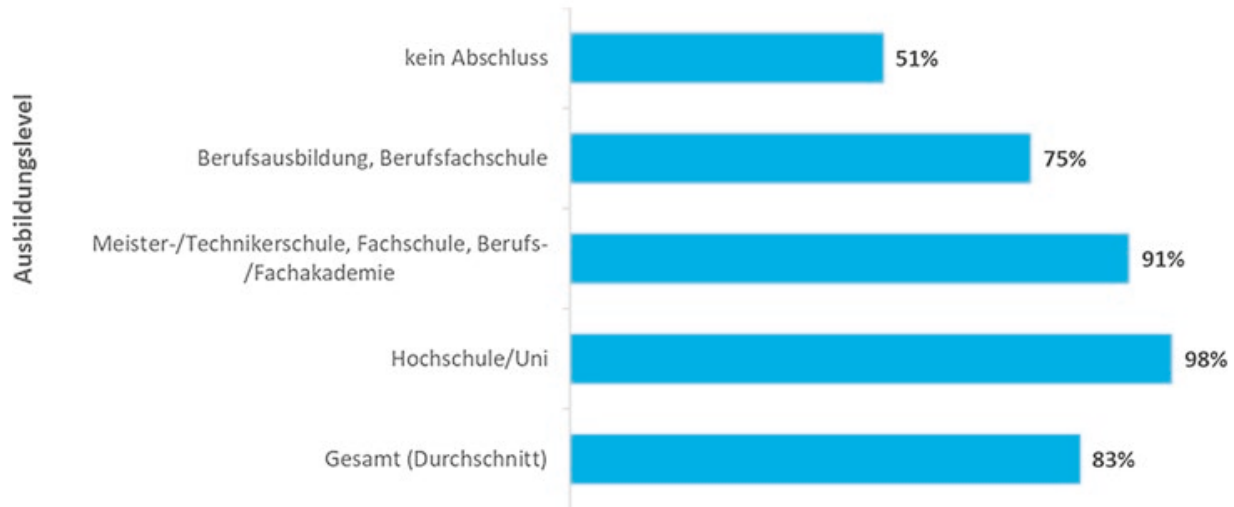

Abb. 2.1 Nutzung digitaler Technologien am Arbeitsplatz nach Ausbildungsniveau

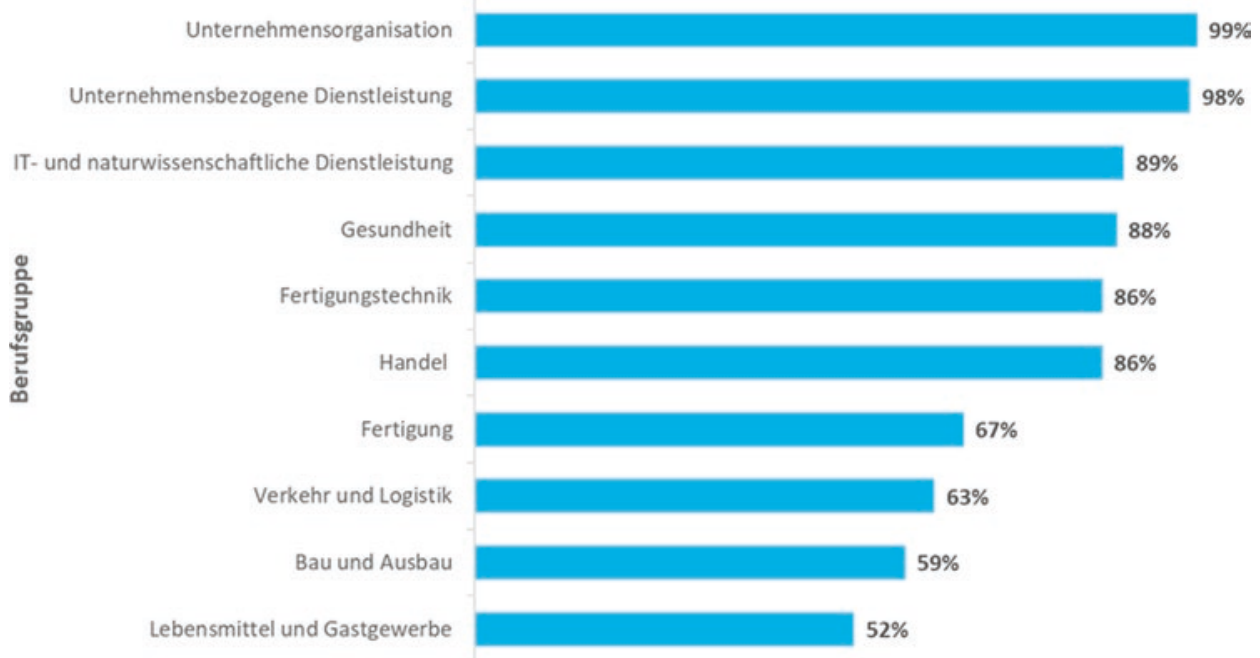

Abb. 2.2 Nutzung digitaler Technologien am Arbeitsplatz nach Berufsgruppe

Unterschiedliche Nutzungsquoten sind zudem nach der beruflichen Stellung im Unternehmen feststellbar. So ist bei Führungskräften die Verbreitung mobil-vernetzter Arbeitsmittel mit einem von der Unternehmensgröße abhängigen Anteil zwischen 61 Prozent und 82 Prozent weitaus größer als im Durchschnitt aller Beschäftigten (14 bis 19 Prozent) (ebd., S. 8). Insgesamt lässt sich feststellen, dass digitalisierte Arbeit vor allem in typischen Büroberufen den Großteil der Tätigkeiten ausmacht und die Nutzung digital-mobiler Arbeitsmittel bei den Beschäftigten insgesamt stark zunimmt. Vor allem in Branchen und 
Tätigkeiten mit wissensintensiven Aufgaben, bei größeren Unternehmen und bei Beschäftigten mit Führungsverantwortung nutzt mittlerweile ein großer Anteil der Arbeitnehmerinnen und Arbeitnehmer mobile digitale Endgeräte für ihre beruflichen Aufgaben.

\subsubsection{Digitale Technologien und Flexibilisierung von Arbeit}

Digitale, mobil-vernetzte Arbeitsmittel sind wichtige „Enabler“ für eine weitreichende räumliche und zeitliche Flexibilisierung von Arbeit, wie sie in vielen Tätigkeitsbereichen zu beobachten ist. Die Arbeit ist nicht mehr örtlich an die Betriebsstätte gebunden und kann auch außerhalb bestehender Betriebszeiten orts- und zeitflexibel erledigt werden. Die Flexibilisierung ist jedoch nicht nur den technischen Möglichkeiten geschuldet. Sie ist auch ein betriebliches Konzept der arbeitsorganisatorischen Modernisierung durch den Abbau hierarchischer Strukturen und der Übertragung von mehr Eigenverantwortung auf die Beschäftigten. Damit sollen zusätzliche Produktivitätsreserven freigesetzt und letztlich eine höhere Innovationsdynamik erreicht werden. Dieses als ,Entgrenzung“ bezeichnete Reorganisationskonzept einer umfassenden Flexibilisierung der Arbeitsverhältnisse ist bereits seit rund zwei Jahrzehnten in der betrieblichen Praxis zu beobachten und Gegenstand einer Vielzahl arbeitssoziologischer Analysen (vgl. Voß 1998; Gottschall und Voß 2003). Entgrenzung von Arbeit findet insbesondere in der zeitlichen und räumlichen Dimension statt. Ausgehend von der traditionellen fordistisch-tayloristisch organisierten Normalarbeit verflüssigt sich die Betriebsförmigkeit von Arbeit mit einer räumlichen Trennung von Produktion und Reproduktion, wenn arbeitsorganisatorische und technische Möglichkeiten den Zugriff auf betriebliche Daten und Kommunikationsprozesse auch von außerhalb des Betriebs, auf Dienstreisen oder zuhause erlauben. Auch die in zeitlicher Hinsicht herkömmlich bestehenden strikten Grenzen zwischen Arbeitszeit und Freizeit erodieren mit einer zunehmenden Flexibilisierung und Pluralisierung von Arbeitszeitregimen (vgl. Kratzer und Sauer 2003, S. 94).

Die räumliche und zeitliche Entgrenzung von Arbeit nur als betriebliches Instrument effizienter Arbeitsorganisation oder Rationalisierung zu betrachten, greift jedoch zu kurz. Flexibles Arbeiten entspricht auch den Wünschen vieler Beschäftigter. Insbesondere Angehörige höherer beruflicher Statusgruppen verbinden mit den neuen technischen und arbeitsorganisatorischen Optionen den Anspruch auf ein selbstbestimmteres Arbeiten und die Hoffnung auf eine bessere Vereinbarkeit von Berufs- und Privatleben. Die Flexibilisierung der Arbeitszeit und des Arbeitsortes ist für diese Gruppe eine wichtige Voraussetzung, um die berufliche Tätigkeit mit familiären Betreuungspflichten und „Sorgearbeit“ besser in Einklang zu bringen. Zudem wandeln sich auch die Ansprüche an die eigene berufliche Arbeit. Dazu gehört auch der Wunsch nach mehr Zeitsouveränität und Selbstbestimmtheit hinsichtlich der Arbeitszeiten und -orte im Rahmen der betrieblichen Erfordernisse (vgl. Bundesministerium für Arbeit und Soziales 2016b, S. 47). Mit der Digitalisierung erhält diese weitreichende Entgrenzung im Raum-Zeit-Gefüge der Arbeitswelt eine neue Dimension, weil die digitale Durchdringung vieler Arbeitsprozesse und die voranschreitende Verbreitung mobil-vernetzter Arbeitsmittel immer mehr Möglichkeiten für 
orts- und zeitflexibles Arbeiten bieten und sich bestehende Grenzen zwischen Betrieb und Wohnung, zwischen Arbeitszeit und Freizeit sowie zwischen Arbeit und Privatleben immer mehr verflüssigen.

\subsubsection{Verbreitung orts- und zeitflexibler Arbeit}

Wenngleich die Digitalisierung als Treiber flexibler Arbeitsformen wirkt, sind hiervon nicht alle Tätigkeiten in gleichem Maße betroffen. Nicht alle Unternehmen nutzen die technischen Möglichkeiten zu einer weiterreichenden Flexibilisierung von Arbeit, ebenso wie nicht alle Beschäftigte sich per se mehr Zeit- und Ortsflexibilität wünschen. Trotz der fortschreitenden Durchdringung der Arbeitsprozesse mit digitalen Technologien ist zudem ein relevanter Teil von Arbeit nicht oder nur sehr eingeschränkt orts- und zeitflexibel zu organisieren, weil die Tätigkeit beispielsweise an Öffnungs- und Betriebszeiten gebunden ist oder ausschließlich im Betrieb oder beim Kunden vor Ort erbracht werden kann. Deshalb ist vor allem die Wissensarbeit als Form geistiger Arbeit, die überwiegend die Generierung von Wissen sowie die Erzeugung, Bearbeitung und Weitergabe von (digitalisierten) Informationen zum Gegenstand hat, besonders geeignet für diese Form der digital-flexiblen Arbeit (vgl. Arlinghaus 2017).

Die Ergebnisse der Arbeitszeitbefragung der Bundesanstalt für Arbeitsschutz und Arbeitsmedizin (BAuA) des Jahres 2017 zeigen, dass die meisten Beschäftigten - trotz Digitalisierung - nur über geringe Zeitautonomie bei der Arbeit verfügen (vgl. Brauner et al. 2018). Rund 40 Prozent können tatsächlich Beginn und Ende ihrer Arbeitszeit bestimmen, wenngleich sich zwei Drittel der Befragten mehr Zeitautonomie bei der Arbeit wünschen (ebd., S. 27). Flexible Arbeitszeitmodelle sind vor allem bei höheren beruflichen Statusgruppen wie Beschäftigten mit Management- und Führungsaufgaben oder bei hochqualifizierten Beschäftigten verbreitet. Arbeitnehmerinnen und Arbeitnehmer, die nicht zu diesen Berufsgruppen gehören, haben in der Regel feste oder nur wenig flexible Arbeitszeiten (vgl. Lott 2017, S. 9). Ebenso ist ein hohes Maß an (selbstbestimmter) Ortsflexibilität der Arbeit nur für einen kleinen Teil der Beschäftigten Realität. Die Mehrheit der Beschäftigten arbeitet an einem festen Arbeitsort, nämlich in der Betriebsstätte des Arbeitgebers, in der auch die Arbeitsmittel (Maschinen, Arbeitsgeräte) und die Arbeitsgegenstände (Werkstücke, Kunden) örtlich gebunden sind (vgl. Brauner et al. 2018). Nach einer Studie des Deutschen Instituts für Wirtschaftsforschung (DIW) sind 40 Prozent der Beschäftigten der Meinung, dass sich ihre Tätigkeit unter Nutzung moderner Informations- und Kommunikationstechnologien auch von zu Hause aus erledigen ließe (Brenke 2016, S. 98).

Dennoch bleibt die Verbreitung von mobiler Arbeit ${ }^{1}$ in Deutschland weit hinter den Wünschen der Beschäftigten sowie den technischen und arbeitsorganisatorischen

\footnotetext{
${ }^{1}$ Mobile Arbeit bezeichnet das Arbeiten außerhalb der Betriebsstätte und umfasst neben der Arbeit von zu Hause (Homeoffice, Telearbeit) auch die Arbeit beim Kunden (Projekt, Service, Vertrieb) oder von unterwegs (Dienstreisen) unter Nutzung digitaler Informations- und Kommunikationsmittel.
} 
Möglichkeiten zurück. Verschiedene Studien beziffern den Anteil von Beschäftigten, die zumindest gelegentlich im Homeoffice arbeiten, zwischen 12 und 17 Prozent (vgl. Brenke 2016; Deutscher Bundestag 2019). Die Sonderauswertung der BAuA-Arbeitszeitbefragung 2017 für den Deutschen Bundestag zeigt, dass die Verbreitung von Arbeit im Homeoffice vor allem von der Branche, der Qualifikation, dem Einkommen und der Haushaltsgröße abhängt. Deutlich über dem Durchschnitt wird mobiles Arbeiten in den Branchen Information und Kommunikation, Finanz- und Versicherungsdienstleistungen sowie freiberufliche/wissenschaftliche und technische Dienstleistungen genutzt. Ebenso haben vor allem Beschäftigte mit Kindern unter 18 Jahren sowie hochqualifizierte und gut Verdienende zu einem höheren Anteil Homeoffice oder Telearbeit mit ihrem Arbeitgeber vereinbart als der Durchschnitt der Beschäftigten.

Die Gründe, (warum die Mehrheit der Beschäftigten nicht von zu Hause oder unterwegs arbeitet, sind vielfältig): Die häufigste Ursache ist die Art der Arbeit (68 Prozent), die eine Tätigkeit nur in der betrieblichen Arbeitsstätte zulässt. Mehr als ein Viertel der Beschäftigten, die nicht ortsflexibel arbeiten, geben als Grund an, dass der Arbeitgeber dies nicht erlaube. Für 15 Prozent ist der persönliche Kontakt zu Kolleginnen und Kollegen wichtiger als der Wunsch, von zu Hause aus zu arbeiten. Es gibt jedoch auch einen relevanten Anteil von Beschäftigten, die sich eine klare Trennung von Berufs- und Privatleben sowie verlässliche und feste Arbeitszeiten wünschen und deshalb nicht orts- oder zeitflexibel arbeiten möchten (vgl. Schicke und Lauenstein 2016, S. 79).

\subsection{Flexibilität des Arbeitsorts und der Arbeitszeit - Wissenschaftliche Erkenntnisse über die Folgen Flexibler Arbeit}

Ein Mehr an Flexibilität von Arbeitsort und -zeit hat sowohl für Arbeitgeber als auch für Arbeitnehmer*innen erhebliche Vorteile. Für Unternehmen dürfte vor allem der flexible Umgang mit Auftragsspitzen sowie die Flexibilität in der Reaktion auf Kundenwünsche und Nachfrageänderungen im Vordergrund stehen (vgl. Bundesanstalt für Arbeitsschutz und Arbeitsmedizin (BAuA) 2017, S. 33). Aber auch das gesteigerte unternehmerische Verantwortungsbewusstsein der Beschäftigten sowie die Steigerung der Attraktivität des Arbeitgebers und die längere Bindung älterer Arbeitnehmer*innen stellen wichtige Argumente dar (vgl. ebd., S. 36, 41). Für Beschäftigte ergeben sich insbesondere Chancen in der Verbesserung der Work-Life-Balance und damit die flexible Organisation ihres Alltags. Zudem führen die Möglichkeiten zu orts- und zeitflexiblem Arbeiten zu einer höheren Arbeitsmotivation sowie -zufriedenheit, das wiederum positiv auf die Gesundheit wirkt (vgl. u. a. Amlinger-Chatterjee 2016; Hanglberger 2010). Darüber hinaus kann das ortsflexible Arbeiten Belastungen reduzieren, die mit arbeitsbedingten Pendelzeiten einhergehen (vgl. Rüger und Ruppenthal 2011, zit. n. Beermann et al. 2018, S. 14). Allerdings zeigen mehrere Untersuchungen, dass die positiven Effekte für die Beschäftigten 
nur dann zum Tragen kommen können, wenn die Flexibilität auch zu einem hohen Grad selbstbestimmt gestaltet werden kann (vgl. hierzu im Überblick Biemann und Weckmüller 2015).

Orts- und zeitflexibles Arbeiten ist jedoch auch mit erheblichen Risiken für die Beschäftigten und damit auch die Unternehmen verbunden. Im Allgemeinen besteht die Gefahr, dass flexible Arbeitsmodelle zu einer erweiterten arbeitsbezogenen Erreichbarkeit führen, was wiederum negativ auf Arbeitsmotivation, Gesundheit und allgemeines Wohlbefinden wirkt (vgl. Arlinghaus und Nachreiner 2014, zit. n. Kauffeld 2019, S. 293; Mellner 2016 zit. n. Kauffeld 2019, S. 293; Sonnentag 2012, zit. n. Kauffeld 2019, S. 293). Insbesondere das ortsflexible Arbeiten kann zu neuen Formen des Präsentismus, längeren Arbeitszeiten, Selbstausbeutung sowie negativer Beeinflussung der Work-Live-Balance führen.

Letztlich wird aus arbeitswissenschaftlicher Perspektive deutlich, dass die zuvor aufgeführten ambivalenten Folgewirkungen von orts- und zeitflexiblem Arbeiten von den Rahmenbedingungen und damit von der Gestaltung und Regulierung der Arbeit abhängig sind. Es besteht somit die Notwendigkeit einer Reduzierung der Belastungsexposition flexibler Arbeitsformen (Beermann et al. 2018, S. 32). Wesentliche Bestandteile einer risikominimierenden und chancenentfaltenden Arbeitsgestaltung sind die Möglichkeit der Mitgestaltung der Beschäftigten, die Festlegung transparenter Rahmenbedingungen und die Reduzierung fremdbestimmter Flexibilisierung.

\subsection{Digital-Flexible Arbeit - Herausforderungen für das Arbeitsrecht}

Die öffentliche Aufmerksamkeit über die Verbreitung digital-flexibler Arbeitsmöglichkeiten sowie die damit einhergehenden arbeitswissenschaftlichen Erkenntnisse in Bezug auf Chancen und Risiken haben eine breite politische und juristische Debatte über Regulierung orts- und zeitflexibler Arbeitsformen sowie über Reformbedarf bestehender Gesetze ausgelöst. Der Kern der Diskussion dreht sich um die Frage, inwieweit Regelungsgegenstand und Regelungszweck vorhandener Rechtsvorschriften den Erfordernissen orts- und zeitflexibler Arbeit entsprechen und ob die Zielsetzung der Rechtsvorschriften mit den vorhandenen Regelungsinstrumenten unter den geänderten Bedingungen in der digitalisierten Arbeitswelt (noch) zu erreichen sind.

\subsubsection{Zeitflexibilität: Diskussionen und potenzieller Reformbedarf}

Bei Vorschlägen zu einer Anpassung des Arbeitsrechts an die Herausforderungen der digitalisierten Arbeitswelt ist nicht nur den vermeintlich erweiterten Flexibilisierungserfordernissen 
Rechnung zu tragen. Es sind vor allem auch die besonderen gesundheitlichen Belastungen und der arbeitsmedizinische Erkenntnisstand zu den Folgen orts- und zeitflexibler Arbeit zu berücksichtigen. Hierbei wird wieder das bereits aufgezeigte Spannungsfeld zwischen arbeitnehmerorientierter selbstbestimmter Flexibilität und unternehmensorientierter Flexibilität sichtbar, das sich auch in der gesellschaftlichen und politischen Debatte und letztlich auch in der rechtswissenschaftlichen Diskussion widerspiegelt.

So manifestiert sich der zuvor skizzierte Flexibilisierungskonflikt in den unterschiedlichen Positionen zum Arbeitszeitrecht. Angesichts der Herausforderungen für die Unternehmen, die mit der Digitalisierung einhergehen, bedarf es nach Meinung der Bundesvereinigung der Deutschen Arbeitgeberverbände (BDA) eines neuen Rechtsrahmens, der die mit Arbeiten 4.0 einhergehenden Chancen für flexibleres Arbeiten und der Entwicklung neuer Geschäftsmodelle eröffnet und nicht beschränkt. Deshalb fordert die BDA die Umstellung der Höchstarbeitszeiten von einer täglichen auf eine wöchentliche Obergrenze und eine erweiterte gesetzliche Grundlage für die Verkürzung von Ruhezeiten per tarifvertraglicher Regelung (vgl. Bundesvereinigung der Deutschen Arbeitgeberverbände (BDA) o. J., S. 5).

Von Gewerkschaftsseite wird der Flexibilisierungsspielraum des geltenden Arbeitszeitgesetzes dagegen als ausreichend betrachtet und kein Änderungsbedarf konstatiert. Der Deutsche Gewerkschaftsbund (DGB) sah seinerseits dagegen die Notwendigkeit, der Flexibilisierung der Arbeitszeit und der zunehmenden Inanspruchnahme der Arbeitnehmerinnen und Arbeitnehmer außerhalb der regulären Dienstzeiten neue Grenzen zu setzen und die Schutzfunktion des Arbeitszeitgesetzes in einer dynamischen Arbeitswelt zu erneuern (DGB Bundesvorstand 2015, S. 4).

Die Enquete-Kommission des Deutschen Bundestags „Internet und digitale Gesellschaft" kam bereits 2013 zu dem Ergebnis, dass die Betriebsparteien und Tarifpartner den negativen Effekten digital erweiterter Erreichbarkeit und Verfügbarkeit durch geeignete Vereinbarungen entgegenwirken sollten. Für mehr Flexibilität im Arbeitszeitgesetz hat sich die Enquete-Kommission nicht ausgesprochen. Auch das Weißbuch Arbeiten 4.0 des Bundesministeriums für Arbeit und Soziales stellt fest, dass der bestehende gesetzliche Rahmen bereits ein hohes $\mathrm{Maß}$ an Flexibilität bietet. „Eine allgemeine Öffnung des Arbeitszeitgesetzes wie eine Abkehr von der Norm des 8-Stundentages zugunsten nur noch einer Wochenhöchstarbeitszeit ist aus Sicht des BMAS mit den Zielen des Arbeitsschutzes und der Zeitsouveränität nicht vereinbar" (Bundesministerium für Arbeit und Soziales (BMAS) 2017, S. 124).

Die Positionierung der politischen Parteien ist in dieser Frage sehr unterschiedlich. Allein die FDP hat sich in ihrer Programmatik für eine Reform des Arbeitszeitgesetzes ausgesprochen, um mehr Flexibilität zu ermöglichen. Die CDU und CSU bleiben hier etwas vage und fordern beispielsweise in ihren Wahlprogrammen zur Bundestagswahl 2017 eine Modernisierung des Arbeitszeitgesetzes, welches zusätzliche Flexibilitätsspielräume für die Tarifpartner eröffnen sollte. Der Verweis auf die EU-Arbeitszeitrichtlinie kann hier als Hinweis verstanden werden, dass die Unionsparteien die tägliche Höchstarbeitszeit des Arbeitszeitgesetzes aufheben und gegen eine wöchentliche Höchstarbeitszeit eintauschen 
wollten. SPD, BÜNDNIS90/Die Grünen und Die Linke sehen keinen Bedarf, das Arbeitszeitgesetz angesichts der Digitalisierung zu flexibilisieren, sondern erachten eher mehr Selbstbestimmung bei der Arbeitszeitgestaltung der Beschäftigten als erforderlich.

\subsubsection{Rechtswissenschaftliche Einordnung des Reformbedarfs Höchstarbeitszeit}

In der rechtswissenschaftlichen Diskussion gibt es einige Autoren, welche die im Arbeitszeitgesetz verankerten täglichen Höchstarbeitszeiten ( 3 ArbZG) als teilweise zu starr beurteilen und eine wöchentliche anstelle einer täglichen Höchstarbeitszeit für sinnvoller erachten (vgl. Jacobs 2016, S. 736). Eine Reihe von Rechtswissenschaftlerinnen und Rechtswissenschaftler beurteilt den Anpassungsbedarf gesetzlicher Vorschriften zu Höchstarbeitszeiten aufgrund neuer Herausforderungen der Digitalisierung eher skeptisch - zumal solche Änderungen alle Beschäftige betreffen würden (vgl. Däubler 2016; Krause 2016; Thüsing 2016). Das Gutachten von Rüdiger Krause „,Digitalisierung der Arbeitswelt - Herausforderungen und Regelungsbedarf“ für den 71. Deutschen Juristentag sieht keinen erkennbaren Grund für eine Öffnung des Acht-Stunden-Tags und erachtet die derzeit möglichen Flexibilisierungsoptionen des $§ 3$ ArbZG als ausreichend, um die wirtschaftlichen Bedürfnisse der Unternehmen zu befriedigen (vgl. Krause 2016, B40).

\section{Ruhezeiten}

Bezüglich der 11-stündigen Ruhezeit zwischen Arbeitsende und Arbeitsbeginn ( $\$ 5$ ArbZG) empfehlen Krause und andere Autoren (vgl. Jacobs 2016, S. 737; Krause 2016, B45) eine konditionierte Öffnung des Arbeitszeitgesetzes für abweichende tarifvertragliche Regelungen, um den gestiegenen Bedürfnissen der Arbeitnehmer nach einer flexibleren Verteilung der individuellen Lage der Arbeitszeit im Sinne einer verbesserten WorkLife-Balance zu entsprechen. Eine unmittelbare Flexibilisierung und Verkürzung von Ruhezeiten durch eine gesetzliche Regelung wäre nach den Vorgaben der EU-Arbeitszeitrichtlinie 2003/88/EG allerdings nicht zulässig. Um mit einer gesetzlichen Öffnung für eine tarifvertraglich abweichende Regelung eine generelle Verkürzung der ununterbrochenen Ruhezeiten zu verhindern, dürfte, so Krause, ,das Ventil allerdings nicht zu weit geöffnet werden“ (Krause 2016, B45). Er plädiert deshalb für eine Beschränkung dieser Ausnahme auf eine bestimmte Anzahl von Fällen pro Jahr (ebd.). Dafür sprechen auch arbeitsmedizinische Erkenntnisse, die die negativen gesundheitlichen Folgen kurzer und nicht zusammenhängender Erholungszeiten belegen, die durch das Arbeiten in ,geteilten Schichten" an den Tagesrandlagen und am Wochenende entstehen.

\section{Unterbrechung von Ruhezeiten}

Wird durch eine stärkere Ortsflexibilität Arbeit ,anytime, anyplace“ ermöglicht, stellt sich bei der mobilen Arbeit außerhalb des Betriebs mithin die Frage, welche Tätigkeiten der Beschäftigten tatsächlich als Arbeitszeit im Sinne des Arbeitszeitgesetzes zu gelten haben. Generell ist Arbeitszeit definiert als ,,der Zeitraum, innerhalb dessen der Arbeitnehmer tatsächlich für den Arbeitgeber arbeitet“ (Baeck et al. 2014, § 2 ArbZG, Rn. 4). Mit der 
dienstlichen Nutzung digital-mobiler Arbeitsmittel wie Smartphones oder Notebooks und geänderten Kommunikationsgewohnheiten kann auch außerhalb der gewöhnlichen Dienstzeiten Arbeit geleistet werden. Meist erfolgt dies jedoch nur in geringfügigem zeitlichem Umfang in Form einer kurzen telefonischen Auskunft und durch das Schreiben einer E-Mail am Abend. Einige Autoren argumentieren, dass es sich hierbei um eine ,nicht nennenswerte Arbeitsleistung" handele, welche den Erholungszweck nicht gefährde und deshalb keine Unterbrechung der Ruhezeiten nach sich ziehe. Um den geänderten Kommunikationsgewohnheiten durch die dienstliche Nutzung von Smartphones Rechnung zu tragen, plädieren einige Autoren für die gesetzliche Klarstellung einer Erheblichkeitsschwelle (vgl. Jacobs 2016, S. 737; Thüsing 2016, S. 98 f.). Ob die gesetzliche Definition einer ,nicht nennenswerten Arbeitsleistung“ rechtssicher anwendbar und in Übereinstimmung mit EU-Recht ist, muss angezweifelt werden. Krause verweist hinsichtlich der Diskussion um die Einführung einer zeitlichen Geringfügigkeitsgrenze bei der Unterbrechung von Ruhezeiten auf die EU-Rechtslage und die bisherige Rechtsprechung des Europäischen Gerichtshofs, die keinen Graubereich zwischen Arbeit und Freizeit vorsieht (Krause 2016, B46-B47).

\section{Erreichbarkeit außerhalb des regulären Arbeitszeitrahmens}

Während das abendliche Schreiben einer E-Mail oder eine kurze telefonische Auskunft außerhalb der Dienstzeiten als Ausnahmefall aus Arbeitsschutzsicht noch als tolerabel gelten dürfte, wachsen sich die geänderten Kommunikationsgewohnheiten durch die dienstliche Nutzung von Smartphones und Notebooks zum Problem aus, wenn sie sich zu einer generell erweiterten Erreichbarkeit der Beschäftigten auch außerhalb der vereinbarten Arbeitszeiten entwickeln. Rund 15 Prozent der Beschäftigten führen täglich oder mehrmals pro Woche in ihrer Freizeit dienstliche Telefonate oder bearbeiten E-Mails. MobilArbeitende werden deutlich häufiger vom Arbeitgeber außerhalb der Arbeitszeit kontaktiert als Beschäftigte, die ausschließlich im Büro arbeiten (vgl. Richter et al. 2017; Schröder 2019) Um dieser Form einer erweiterten bzw. ständigen Erreichbarkeit der mit mobilen Endgeräten ausgestatteten Arbeitnehmerinnen und Arbeitnehmer Schranken zu setzen, empfiehlt Krause, diesen Tatbestand als Rufbereitschaft zu qualifizieren und diese Zeiten ,auf eine bestimmte Anzahl von Tagen im Monat zu beschränken, um zu verhindern, dass sich ein Arbeitnehmer praktisch kontinuierlich entweder im Arbeitsmodus oder im Erreichbarkeits- bzw. Rufbereitschaftsmodus befindet“" (Krause 2016, S. B46).

Ein von den Gewerkschaften gefordertes „Recht auf Nichterreichbarkeit“ zum Schutz der Beschäftigten vor einer Kontaktaufnahme außerhalb der Dienstzeiten durch Vorgesetzte, Kollegen und Kunden lässt sich im Rahmen des Mitbestimmungsrechts des Betriebsrats dadurch realisieren, dass bestimmte Zeiten von der Rufbereitschaft und einer geforderten Erreichbarkeit durch Betriebs- oder Dienstvereinbarung ausgeklammert werden (vgl. Däubler 2016, S. 19). Den Handlungsbedarf sieht auch das Bundesarbeitsministerium auf der betrieblichen Ebene und nicht beim Gesetzgeber und verweist auf eine Reihe von Betriebs- und Dienstvereinbarungen zur Nichterreichbarkeit (vgl. Bundesministerium für Arbeit und Soziales (BMAS) 2017, S. 119). Bereits nach jetziger Rechtslage 
hat der Arbeitgeber kein Recht, den Arbeitnehmer in arbeitsfreien Zeiten außerhalb der regulären Arbeitszeit am Abend oder am Wochenende in Anspruch zu nehmen. Dies im Arbeitszeitgesetzt ausdrücklich festzuschreiben, könnte dem bestehenden Rechtszustand allerdings mehr „Nachdruck verleihen“ (vgl. Däubler 2016, S. 19).

\section{Sonntagsarbeit}

Die verschiedentlich geforderte Zulässigkeit der (digitalen) häuslichen bzw. mobilen Arbeit an Sonn- und Feiertagen ist nach Einschätzung von Krause ,im Grundsatz nicht statthaft, auch wenn sie den Arbeitnehmer nur geringfügig in Anspruch nimmt und für außenstehende Dritte nicht wahrnehmbar ist" (Krause 2016, S. B48). Das gleiche gilt für die Anforderung einer Erreichbarkeit der Beschäftigten an Sonn- und Feiertagen. Solche Überlegungen zur Freigabe von Sonn- und Feiertagsarbeit als Maßnahme zur verbesserten Vereinbarkeit von Beruf und Familie dürften regelmäßig die „Sogwirkung unterschätzen, die durch die Möglichkeit ,freiwilliger' Arbeit geschaffen wird und die den Sonn- und Feiertagsschutz auf breiter Front aushöhlen würde“" (ebd.).

Bei allen Reformvorschlägen zum Arbeitszeitgesetz ist zu berücksichtigen, dass hiervon auch die Mehrheit von Beschäftigten, die in traditionellen Arbeitsformen tätig sind und somit nicht überwiegend selbstbestimmt orts- und zeitflexibel arbeiten, von solchen Änderungen erfasst würde. Für diese würde eine Erhöhung der täglichen Höchstarbeitszeit und eine weitgehende Verkürzung von Ruhezeiten ,einen schlichten Sozialabbau bedeuten, dem keine Produktivitätsgewinne gegenüberstehen. Helfen kann hier nur eine differenzierende Lösung, die am besten auf betrieblicher Ebene anzusiedeln wäre“ (Däubler 2016, S. 19). Auch Thüsing hält angesichts der Herausforderungen der Digitalisierung in der Arbeitswelt den Ruf nach dem Gesetzgeber allenfalls im Sinne einer Präzisierung der geltenden Arbeitszeitvorschriften für erforderlich und empfiehlt eine flexible und den Arbeitsschutz wahrende Gestaltung auf der betrieblichen Ebene innerhalb des geltenden Rahmen des Arbeitszeitgesetzes (vgl. Thüsing 2016, S. 98 f.).

\subsubsection{Ortsflexibilität: Diskussion und potenzieller Reformbedarf}

\subsubsection{Positionen und Diskussionen zum Reformbedarf der Flexibilität des Arbeitsorts}

Zuvor wurde bereits aufgezeigt, dass ein räumlich selbstbestimmtes Arbeiten für viele Beschäftigte noch weit von der Realität entfernt ist. Fraglos erweitern digitale Technologien die Möglichkeiten der Ortsflexibilität, dennoch gib es nach wie vor eine große Lücke zwischen Wunsch und Wirklichkeit der Beschäftigten, neben Lage und Verteilung der Arbeitszeit auch den Arbeitsort mitbestimmen zu können.

Von Gewerkschaftsseite wird vor diesem Hintergrund ein „Recht auf Homeoffice“ für ein selbstbestimmtes mobiles Arbeiten gefordert, welches als gesetzlicher Rechtsanspruch zu normieren und durch Tarifvertrag und betriebliche Regelungen auszugestalten ist (vgl. DGB-Bundesvorstand 2019, S. 2). Die Bundesvereinigung der Deutschen Arbeitgeberverbände hingegen 
lehnt ein solches Ansinnen ab. Der Arbeitgeberverband verweist auf den hohen Anteil von Unternehmen, die ihren Beschäftigten bereits mobiles Arbeiten ermöglichen und befürchtet u. a. eine Spaltung der Belegschaften, da es eine Reihe von Beschäftigtengruppen gibt, wie Pflegekräfte oder Kraftfahrer, die aufgrund ihrer Tätigkeit von einem solchen Rechtsanspruch ausgeschlossen blieben (Bundesvereinigung der Deutschen Arbeitgeberverbände (BDA) 2019).

Während in der Parteienlandschaft SPD, Die Linke und BÜNDNIS 90/Die Grünen für mehr Selbstbestimmung der Beschäftigten bei der Frage des Arbeitsorts eintreten, lehnen CDU, CSU und FDP einen gesetzlichen Anspruch auf mobiles Arbeiten bislang ab. Die Linke betont in der Diskussion, dass „Homeoffice (...) kein gesellschaftlicher Gewinn werden [kann], wenn die Beschäftigten dabei ausbrennen“ (Die Linke 2019) und fordert „gesetzliche Leitplanken“ für diese ortsflexible Arbeitsform. Die Enquete-Kommission des Deutschen Bundestags sprach sich bereits 2013 mehrheitlich für die Förderung von Arbeit im Homeoffice aus, aber schränkte dies zugleich ein, sodass bei den möglicherweise erforderlichen gesetzlichen Maßnahmen zur Förderung mobilen Arbeitens und zur Verbesserung der Work-Life-Balance die letzte Entscheidung darüber, „ob und wieweit Tätigkeiten an einem von dem Beschäftigten selbst zu bestimmenden Arbeitsplatz erbracht werden dürfen (...), der unternehmerischen Freiheit vorbehalten bleiben (soll)“ (Deutscher Bundestag 2013, S. 99).

In vielen, meist größeren Unternehmen besteht ein Anspruch der Beschäftigten auf mobiles Arbeiten im Homeoffice auf Grundlage eines Tarifvertrags oder einer betrieblichen Vereinbarung. Um die Verbreitung mobiler Arbeit über Großunternehmen hinaus zu fördern, will das Bundesministerium für Arbeit und Soziales prüfen, ,einen gesetzlichen Anspruch auf mobile Arbeit zu schaffen, den der Arbeitgeber z. B. aus betrieblichen Gründen ablehnen kann“ (Bundesministerium für Arbeit und Soziales (BMAS) 2019, S. 21). Der Bundeswirtschaftsminister erachtet hingegen einen solchen Rechtsanspruch zur Förderung mobilen Arbeitens als nicht erforderlich (Drebes und Quadbeck 2019).

\subsubsection{Rechtswissenschaftliche Einordnung des Reformbedarfs}

Das durch die Digitalisierung verstärkt mögliche ortsflexible Arbeiten wirft - wie dargestellt - zahlreiche Fragen hinsichtlich des Reformbedarfs des Arbeitsschutzgesetzes und der darunter gefassten Verordnung auf. Adressiert werden im Folgenden der vielfach geforderte Rechtsanspruch auf mobiles Arbeiten, die Anforderungen des Arbeitsschutzes bei mobiler Arbeit sowie die Anwendung des Bildschirmarbeitsrechts bei mobiler Arbeit.

\section{Rechtsanspruch auf mobiles Arbeiten}

In der rechtswissenschaftlichen Diskussion um einen Rechtsanspruch auf mobiles Arbeiten schlagen mehrere Autoren eine Regelung analog zum Teilzeit- und Befristungsgesetz (TzBfG) vor, um die Ortssouveränität der Beschäftigten zu stärken (vgl. Schwemmle und Wedde 2012; Krause 2016; Thüsing 2016). Auf einer solchen gesetzlichen Grundlage könnten Arbeitnehmerinnen und Arbeitnehmer verlangen, zeitweise im Homeoffice zu arbeiten, sofern keine betrieblichen Gründe entgegenstehen. Ablehnungsgründe wären demnach eine wesentliche Beeinträchtigung der Organisation, des Arbeitsablaufs oder der 
Sicherheit im Betrieb oder wenn durch das Homeoffice unverhältnismäßige Kosten verursacht würden. Gemeinsam mit Thüsing schlägt Krause in seinem Gutachten für den 71. Deutschen Juristentag vor, mit einer Stärkung der Selbstbestimmung über den Arbeitsort auch eine höheren Souveränität der Beschäftigten hinsichtlich der Lage und Verteilung der Arbeitszeit gesetzlich zu verankern, um damit die positiven Wirkungen orts- und zeitflexibler Arbeit sowohl für die Beschäftigten wie auch für die Unternehmen zu stärken (vgl. Krause 2016, B48). Eine solche Stärkung einer selbstbestimmten orts- und zeitflexiblen Arbeit führt nicht nur zur Verbesserung der Work-Life-Balance der Beschäftigten, sondern verbessert auch die subjektive Belastungsverarbeitung und wirkt somit als wirksamer Belastungspuffer für eine mögliche psychische Beanspruchung, die mit mehr Flexibilität einhergehen kann.

\section{Anwendung der Vorschriften der Arbeitsstättenverordnung bei mobiler Arbeit}

Im Jahr 2016 hat die Bundesregierung nach einer intensiven politischen Debatte die Arbeitsstättenverordnung reformiert und die Bildschirmarbeitsverordnung in diese Vorschrift integriert. Angesichts der wachsenden Bedeutung und Verbreitung ortsflexibler Arbeitsformen wurden die konkreten Anforderungen für Telearbeitsplätze und „wie diese Arbeitsplätze außerhalb des Betriebes zum Schutz der Beschäftigten zu gestalten sind“ (Bundesrat 2016, S. 34), neu geregelt. Nach der Reform der ArbStättV findet diese nunmehr auch Anwendung auf „Telearbeitsplätze“, bei denen es sich gemäß § 2 Abs. 7 ArbStättV um fest eingerichtete Bildschirmarbeitsplätze im Privatbereich der Beschäftigten handelt. Für Telearbeit als außerbetriebliche Arbeitsform gelten jedoch nur die Vorschriften der Arbeitsstättenverordnung zur Gefährdungsbeurteilung ( 3 ArbStättV) und zur Unterweisung der Beschäftigten ( $\$ 6$ ArbStättV) sowie die Regelungen zur Bildschirmarbeit (Anhang Nr. 6 ArbStättV) - und auch diese nur mit weiteren Einschränkungen und Vorbehalten.

Mit der in der Arbeitsstättenverordnung definierten „Telearbeit“ wird jedoch nur eine in der betrieblichen Praxis eher wenig angewandte Variante der ortsflexiblen Arbeit außerhalb des Betriebs von der Arbeitsschutzvorschrift erfasst - nämlich die eines vertraglich vereinbarten und vom Arbeitgeber fest eingerichteten Telearbeitsplatzes in der Wohnung der Beschäftigten. Nicht unter den Geltungsbereich der ArbStättV fällt die aktuell eher verbreitete Form ortsflexibler Arbeit, bei der das Unternehmen in Absprache mit den Vorgesetzten oder auf Grundlage einer betrieblichen Regelung auf die tägliche Anwesenheit der Beschäftigten im Betrieb verzichtet. Die Beschäftigten können bei dieser Form ortsflexibler Arbeit einen Teil ihrer Arbeit zu Hause, unter Nutzung des dienstlichen Notebooks und Mobiltelefons im „Homeoffice“ erledigen, ohne dass der Arbeitgeber hierfür einen häuslichen Arbeitsplatz eingerichtet hat. Ebenso wenig unterliegt der Arbeitsstättenverordnung auch das mobile Arbeiten auf Dienstreisen oder beim Kunden (vgl. ebd., S. 36).

\section{Anforderungen an den Arbeitsschutz bei mobiler Arbeit}

Auch wenn sich die Anwendung der Arbeitsstättenverordnung nur auf „Telearbeit“ beschränkt, ist es unstrittig, dass vom rechtlich übergeordneten Arbeitsschutzgesetz alle 
Formen von (mobiler) Arbeit erfasst werden. Dies bestätigt auch die Bundesregierung in einer Antwort auf eine Bundestagsanfrage: „Das Arbeitsschutzgesetz gilt grundsätzlich in allen Tätigkeitsbereichen und findet auch bei orts- und zeitflexibler Arbeit Anwendung. Der Arbeitgeber hat mögliche Gefährdungen für die Gesundheit und Sicherheit der Beschäftigten zu ermitteln und die notwendigen Schutzmaßnahmen zu treffen" (Deutscher Bundestag 2019, S. 9). Dass der Gesundheitsschutz bei ortsflexibler Arbeit schwieriger zu organisieren und zu implementieren ist, ändert nichts an der Pflicht des Arbeitgebers zur Einhaltung der rechtlichen Vorschriften und arbeitswissenschaftlichen Standards zum Arbeitsschutz. Gemäß $\S \S 3$ ff. ArbSchG obliegt dem Arbeitgeber generell die Beachtung grundlegender Arbeitsschutzpflichten zur Gewährleistung von Sicherheit und Gesundheit der Beschäftigten bei der Gestaltung ihrer Arbeit. Der Arbeitgeber hat die Pflicht, die erforderlichen Maßnahmen des Arbeitsschutzes unter Berücksichtigung der Umstände zu treffen, die Sicherheit und Gesundheit der Beschäftigten bei der Arbeit beeinflussen.

Die hierzu zu ergreifenden Schutzmaßnahmen bei ortsflexiblem Arbeiten können von der Bereitstellung mobiler, ergonomisch gestalteter Arbeitsmittel über regelmäßige Unterrichtung und Unterweisung der Arbeitnehmer hinsichtlich möglicher Gefährdungspotenziale und einzuhaltender Schutzmaßnahmen bis hin zum Verbot der Arbeitsleistung unter für den Arbeitnehmer erkennbar gesundheitsgefährdenden Umständen reichen (vgl. Deutscher Bundestag 2017, S. 9). Trotz der analogen Übertragungsmöglichkeit der Arbeitsstättenverordnung auf ortsflexibles Arbeiten besteht weiterhin rechtliche Unklarheit bezüglich der Anwendung des Bildschirmarbeitsrechts und einer Konkretisierung des Arbeitsschutzrechts bei Tätigkeiten, die am Notebook oder unter Nutzung von Tablets und Smartphones außerhalb des betrieblichen Arbeitsplatzes erledigt werden.

\section{Anwendung des Bildschirmarbeitsrechts}

Rechtsunsicherheit bei der Anwendung der Arbeitsstättenverordnung ergibt sich zudem bei der Unterscheidung zwischen Telearbeit nach der Definition der Arbeitsstättenverordnung und der überwiegend angewandten Praxis der regelmäßigen Arbeit im Homeoffice, die mit dem Arbeitgeber oftmals auch nur mündlich vereinbart ist. Inwieweit eine regelmäßige und ganztägige Arbeit im Homeoffice unter Nutzung von Notebooks ohne externe Eingabegeräte den Arbeitsschutznormen und den Vorgaben von Ziffer 6.4. Anhang 6 ArbStättV entspricht, wonach solche Geräte an Arbeitsplätzen nur kurzzeitig genutzt werden dürfen, ist zumindest strittig und muss im Einzelfall im Rahmen einer Gefährdungsbeurteilung bewertet werden. Grundsätzlich kann der Arbeitgeber aufgrund seiner allgemeinen Schutzpflichten gemäß $\S 3,4$ ArbSchG eine regelmäßige Arbeit an einem Arbeitsplatz in der Wohnung der Beschäftigten nur zulassen, wenn gewährleistet ist, dass damit keine Gefahren für die Sicherheit und die Gesundheit der Arbeitnehmerinnen und Arbeitnehmer ausgehen. Die Erfüllung von Grundanforderungen an eine ergonomische Arbeitsplatzgestaltung muss deshalb auch bei der Arbeit im „Homeoffice“ der Beschäftigten gewährleistet sein. 


\subsection{Resümee: Regelungsbedarf im Kontext Orts- und Zeitflexibler Arbeit}

Die Ausführungen in diesem Beitrag zeigen, dass die Digitalisierung mit ihren erhöhten Möglichkeiten für orts- und zeitflexibles Arbeiten zu einer deutlichen Veränderung der Arbeit führt. Zwar arbeitet nach derzeitigem Stand nur ein eher kleiner, wenngleich steigender Teil der Beschäftigten örtlich und zeitlich flexibel. Dabei können meist eher hochqualifizierte Beschäftigte in wissensintensiven Bereichen orts- und zeitflexibel arbeiten und von den Vorteilen von mehr Arbeitsautonomie, einer besseren Vereinbarkeit von Arbeit und Privatem und einer höheren Arbeitsmotivation sowie -zufriedenheit profitieren. Jedoch wünscht sich ein weitaus größerer Teil der Beschäftigten mehr zeitliche Flexibilität bei der Arbeit und die Möglichkeit, im Homeoffice zu arbeiten - letzteres scheitert jedoch nicht selten am Arbeitgeber, der eine höhere Autonomie der Beschäftigten bei der Wahl des Arbeitsorts nicht zulässt.

Orts- und zeitflexibles Arbeiten kann jedoch auch negative Folgen für die Beschäftigten haben. In vielen Fällen zeigt sich eine Ausdehnung der Arbeitszeiten infolge flexibler Arbeitsmodelle, einer Verschiebung von Arbeitszeiten in die Randlagen des Tages und auf das Wochenende sowie eine erweiterte Erreichbarkeit auch außerhalb regulärer Dienstzeiten. Die Analysen der Auswirkungen dieser Veränderungen auf Wohlbefinden und Gesundheit zeigen ein klares Ergebnis: Die einerseits positiven Wirkungen erweiterter Autonomie und verbesserter Work-Life-Balance drohen durch eine erhöhte Arbeitsintensität und steigende psychische Belastungen überkompensiert zu werden. Insbesondere ein hohes Maß an fremdbestimmter Flexibilität verstärkt nicht nur die Belastungssituation, sondern führt gleichzeitig dazu, dass aufgrund des eingeschränkten Handlungsspielraums eine positive subjektive Belastungsverarbeitung durch mehr Arbeitsautonomie nicht erfolgen kann.

Aus Sicht der Arbeitswissenschaft ergibt sich die Notwendigkeit, die Belastungsexposition flexibler Arbeitsformen zu reduzieren sowie lange Arbeitszeiten und kurze Ruhezeiten zu vermeiden. Zudem sollten der Handlungsspielraum der Beschäftigten erweitert werden, um somit die subjektive Belastungsverarbeitung zu verbessern. Letztlich besteht die Herausforderung der Arbeitsregulierung im Ausgleich zwischen einem Mehr an Flexibilität und Selbstbestimmung einerseits sowie der Vermeidung von Selbstüberforderung und der Gewährleistung eines effektiven Arbeits- und Gesundheitsschutzes auf der anderen Seite.

Nachfolgend zeigen wir auf, welche Handlungsfelder bzw. -erfordernisse mit Blick auf die Regulierung flexibler Arbeitsformen besteht. Eine ausführliche Darstellung findet sich bei Daum und Zanker (2020).

\section{Arbeitszeitgesetz: ausreichenden Arbeitsschutz erhalten}

Das Arbeitszeitgesetz bietet bereits ausreichende Flexibilitätsspielräume für tägliche Arbeitszeiten von bis zu zehn Stunden und eröffnet bereits die Möglichkeit einer Verkürzung von Ruhezeiten auf Grundlage eines Tarifvertrages, sofern dies die Arbeit erfordert. Der Wunsch nach mehr Flexibilisierung der Arbeitszeit mag aus Sicht der Unternehmen nachvollziehbar sein. Es besteht keine Notwendigkeit nach einer weiteren Flexibilisierung des 
gesetzlichen Arbeitszeitrahmens, die aus der Option für mehr orts- und zeitflexible Arbeit in der digitalisierten Welt resultiert. Es besteht eher der Bedarf nach effektiverem Arbeitsschutz, der durch die verstärkte Orts- und Zeitflexibilität gefährdet sein kann. Ein Recht auf Nichterreichbarkeit, umgesetzt und kontrolliert auf der betrieblichen Ebene, wird beispielsweise als allgemein erforderlich erachtet. Zudem könnte im Arbeitszeitgesetz klargestellt werden, dass der Arbeitgeber dafür Sorge zu tragen hat, dass Arbeitnehmer*innen außerhalb ihrer regulären Arbeitszeit sowie von wirksam angeordneten bzw. vereinbarten Rufbereitschaftszeiten grundsätzlich nicht in dienstlichen Belangen kontaktiert werden.

\section{Mehr selbstbestimmtes Arbeiten ermöglichen}

Ein Rechtsanspruch auf mobiles Arbeiten könnte einen Beitrag leisten, mehr Beschäftigten eine höhere Ortssouveränität zu ermöglichen. Die dargestellten Möglichkeiten einer gesetzlichen Grundlage analog zum Teilzeit- und Befristungsgesetz zu schaffen, sind praktikabel und ausgewogen zugleich. Somit würde ein grundsätzlicher Rechtsanspruch auf Homeoffice eröffnet, sofern sich die Arbeit hierzu eignet und keine betrieblichen Gründe dagegenstehen. Die Ausgestaltung muss dann über einen Tarifvertrag oder eine betriebliche Vereinbarung erfolgen.

\section{Rechtliche Klarstellung der Anwendung der Arbeitsschutzvorschriften bei mobiler und ortsflexibler Arbeit}

Der Gesetzgeber sollte die dargelegte bestehende Regelungslücke bei ortsflexibler Arbeit schließen bzw. rechtlich klarstellen, denn der Rückschluss, dass beim mobilen Arbeiten von unterwegs oder im Homeoffice der gesetzliche Arbeitsschutz nicht zu beachten sei oder gesetzliche Vorschriften keine Anwendung fänden, ist falsch. Es gibt keinen Dissens in der Einschätzung, dass das Arbeitsschutzgesetz als die zentrale Vorschrift zum Gesundheitsschutz der Arbeitnehmerinnen und Arbeitnehmer auf jede Form mobiler Arbeit anzuwenden ist. Es herrscht jedoch zum Teil Rechtsunsicherheit, was die konkrete analoge Anwendbarkeit der Arbeitsstättenverordnung und der Bildschirmarbeitsvorschriften auf ortsflexible Arbeitsformen außerhalb der Telearbeit betrifft. Hier ist eine rechtliche Klarstellung erforderlich.

\section{Mobile Arbeit kollektivvertraglich regeln}

Viele Regulierungsfelder orts- und zeitflexibler Arbeit sind nicht (nur) auf der staatlichen Ebene zu verorten, sondern liegen im Zuständigkeitsbereich der Tarifvertrags- und Betriebsparteien. So sind sowohl Lage und Verteilung der Arbeitszeit, Erreichbarkeits- und Nichterreichbarkeitszeiten, konkrete Maßnahmen des Arbeits- und Gesundheitsschutzes sowie die in diesem Beitrag nicht thematisierten Fragen der Qualifizierung und des Datenschutzes bei orts- und zeitflexibler Arbeit in betriebsnahen Vereinbarungen zu regeln. Mobile Arbeit ist eine der Arbeitsformen, die sich wirksam auf der betrieblichen Ebene umsetzen und kontrollieren ließen. Der zwischen ver.di und Deutsche Telekom im Juni 2016 abgeschlossene Tarifvertrag zu „Mobile Working“ kann hier als Beispiel gelten. Die Vereinbarung regelt die wesentlichen Eckpunkte von mobiler Arbeit bei der Deutschen Telekom und enthält an verschiedenen Stellen explizite Aufträge für die betriebliche Ausgestaltung (vgl. Zanker 2017). 


\section{Arbeitszeit- und Arbeitsschutzrecht als Konfliktfeld digitaler Arbeitspolitik}

In unserem Beitrag und weiteren angeführten Analysen wird deutlich, dass das Arbeitszeitund Arbeitsschutzrecht eines der Konfliktfelder arbeitspolitischer Akteure ist. Arbeitsrechtliche Weiterentwicklungen im Sinne von Beschäftigten und Unternehmen sucht man vergebens. Schwemmle und Wedde (2018) ziehen deshalb auch das eher ernüchternde Resümee einer weitgehenden Ergebnislosigkeit der Arbeitspolitik angesichts der vielfältigen Herausforderungen, die mit der Digitalisierung einhergehen (Schwemmle und Wedde 2018, S. 48). Unter anderem sei es der Politik in den ,,vergangenen Jahren (...) nicht gelungen, offenkundig notwendige arbeitsrechtliche Anpassungsschritte durchzuführen“ (ebd.), die den Arbeitnehmerinnen und Arbeitnehmern auch in der digitalisierten Welt die bisherigen Standards von Arbeitsschutz und Qualität der Arbeitsbedingungen sicherstellen.

Zwei Jahre später lässt sich konstatieren, dass dieser „offenkundig notwendige“ Anpassungs- und Konkretisierungsbedarf im Arbeitsrecht weiterhin besteht. Die Politik konnte sich bislang zu keiner Reform der entsprechenden arbeitsrechtlichen Vorschriften durchringen, obwohl eine Reihe von konkreten Regelungsvorschlägen, wie hier gezeigt, vorliegen. Einige politische Initiativen befinden sich seit geraumer Zeit im Stadium politischer Ankündigung, wie das Beispiel des Anspruchs auf Homeoffice oder die Konkretisierungen der rechtlichen Anforderungen an den Arbeitsschutz bei mobiler Arbeit zeigen. Dass bislang daraus keine gesetzgeberischen Initiativen entstanden sind, dürfte vor allem daran liegen, dass viele der hier analysierten Handlungsfelder bei der Regulierung orts- und zeitflexibler Arbeit innerhalb der Regierungskoalition und zwischen Arbeitsgeberverbänden und Gewerkschaften politisch äußerst strittig sind. Der politische Kompromiss zwischen unterschiedlichen Auffassungen zum arbeitsrechtlichen Anpassungsbedarf scheint daher die Bewahrung des Status quo zu sein.

\section{Literatur}

Amlinger-Chatterjee, M. (2016). Psychische Gesundheit in der Arbeitswelt. Atypische Arbeitszeiten. Dortmund/Berlin/Dresden: Bundesanstalt für Arbeitsschutz und Arbeitsmedizin (BAuA).

Arlinghaus, A., \& Nachreiner, F. (2014). Health effects of supplemental work from home in the European Union. Chronobiology International, 31(10), 1100-1107.

Arlinghaus, A. (2017). Wissensarbeit. Aktuelle arbeitswissenschaftliche Erkenntnisse. Düsseldorf: Hans-Böckler-Stiftung.

Baeck, U., Deutsch, M., \& Kramer, N. (2014). Arbeitszeitgesetz. Kommentar, 3. Aufl. München: Beck-Online. https://beck-online.beck.de/?vpath=bibdata/komm/BaeckDeutschArbZG_3/cont/ BaeckDeutschArbZG.htm.

Bundesanstalt für Arbeitsschutz und Arbeitsmedizin. (BAuA). (Hrsg.). (2017). Flexible Arbeitszeitmodelle. Überblick und Umsetzung. Dortmund/Berlin/Dresden.

Bundesministerium für Arbeit und Soziales (BMAS). (Hrsg.). (2019). Zukunftsdialog Neue Arbeit - Neue Sicherheit. Ergebnisbericht. Berlin.

Bundesvereinigung der Deutschen Arbeitgeberverbände (BDA). (2019). Mobiles Arbeiten nur auf freiwilliger Basis. https://www.arbeitgeber.de/www/arbeitgeber.nsf/id/8DD4C0B6B7C463 82C1258402003FBEEF. Zugegriffen am 26.06.2019.

Bundesvereinigung der Deutschen Arbeitgeberverbände (BDA). (Hrsg.). (o. J.). New Work. Zeit für eine neue Arbeitszeit. Berlin. 
Beermann, B., Amlinger-Chatterjee, M., Brenscheidt, F., Gerstenberg, S., Niehaus, M., \& Wöhrmann, A. M. (2018). Orts- und zeitflexibles Arbeiten: Gesundheitliche Chancen und Risiken (2. Aufl.). Dortmund/Berlin/Dresden: Bundesanstalt für Arbeitsschutz und Arbeitsmedizin (BAuA) (Hrsg.). Biemann, T., \& Weckmüller, H. (2015). Effektives Arbeiten, wann und wo man will? Home-OfficeAngebote erhöhen Arbeitszufriedenheit und Arbeitgeberattraktivität, Vertrauensarbeitszeit wirkt zudem produktivitätssteigernd. Personal Quarterly, 67, 46-49.

Bundesministerium für Arbeit und Soziales (BMAS). (Hrsg.). (2016a). Monitor: Digitalisierung am Arbeitsplatz. Aktuelle Ergebnisse einer Betriebs- und Beschäftigtenbefragung. Berlin.

Bundesministerium für Arbeit und Soziales (BMAS). (Hrsg.). (2016b). Wertewelten Arbeiten 4.0. Berlin. Bundesministerium für Arbeit und Soziales (BMAS). (Hrsg.). (2017). Weißbuch Arbeiten 4.0. Berlin.

Brauner, C., Wöhrmann, A. M., \& Michel, A. (2018). BAuA-Arbeitszeitbefragung: Arbeitszeitwünsche von Beschäftigten in Deutschland. Dortmund/Berlin/Dresden: Bundesanstalt für Arbeitsschutz und Arbeitsmedizin (BAuA) (Hrsg.).

Brenke, K. (2016). Home Office: Möglichkeiten werden bei weitem nicht ausgeschöpft. DIW Wochenbericht, 5, 95-104.

Bundesrat. (Hrsg.). (2016). Entwurf einer Verordnung zur Änderung von Arbeitsschutzverordnungen. Verordnungsentwurf des Bundesrates. Berlin (Drucksache, 506/16).

Däubler, W. (2016). Digitalisierung und Arbeitsrecht. Abhandlung. In Soziales Recht (6, Sonderausgabe).

Daum, M., \& Zanker, C. (2020). Digitale Arbeitswelt - vernetzt, flexibel und gesund? Status quo und Perspektiven der Gestaltung und Regulierung von Orts- und Zeitflexibilität. Arbeitspapier. https://www.input-consulting.de/files/inpcon-DATA/download/2020_Regulierung_orts-zeitflexibles-Arbeiten_Transwork_INPUTConulting.pdf. Zugegriffen am 23.03.2020.

Deutscher Bundestag. (Hrsg.). (2013). Achter Zwischenbericht der Enquete-Kommission „Internet und digitale Gesellschaft“. Wirtschaft, Arbeit, Green IT. Berlin (Drucksache, 17/12505).

Deutscher Bundestag. (Hrsg.). (2017). Telearbeit und Mobiles Arbeiten. Voraussetzungen, Merkmale und rechtliche Rahmenbedingungen (Sachstand, WD 6 - 3000 - 149/16).

Deutscher Bundestag. (Hrsg.). (2019). Homeoffice: Stand, Chancen und Risiken für Arbeitnehmerinnen und Arbeitnehmer. Antworten der Bundesregierung auf die kleine Anfrage der Abgeordneten Jessica Tatti, Susanne Ferschl, Matthias W. Birkwald, weiterer Abgeordneter und der Fraktion DIE LINKE. - Drucksache 19/8494 - (Drucksache 19/9032 (03.04.2019)).

DGB-Bundesvorstand. (Hrsg.). (2015). Digitalisierung der Arbeitswelt. Kommentar des DGB-Bundesvostands zum Positionspapier der Bundesvereinigung der Deutschen Arbeitgeberverbände (BDA) zur Digitalisierung von Wirtschaft und Arbeitswelt. Berlin.

DGB-Bundesvorstand. (Hrsg.). (2019). Diskussionspapier des DGB für einen gesetzlichen Ordnungsrahmen für selbstbestimmtes mobiles Arbeiten. Berlin

Die Linke. (2019). Viel heiße Luft, wenig Verbesserung bei Heils „Arbeit von morgen“-Vorschlägen. Pressemitteilung von Jessica Tatti, 20. September 2019. https://www.linksfraktion.de/presse/ pressemitteilungen/detail/viel-heisse-luft-wenig-verbesserung-bei-heils-arbeit-von-morgen-vorschlaegen/. Zugegriffen am 26.01.2020.

Drebes, J., \& Quadbeck, E. (2019). Ein Rechtsanspruch auf Homeoffice ist nicht notwendig. Interview mit Wirtschaftsminister Peter Altmaier. https://rp-online.de/politik/deutschland/wirtschaftsminister-peter-altmaier-ein-rechtsanspruch-auf-homeoffice-ist-nicht-notwendig_aid-37327077. Zugegriffen am 13.09.2019.

Gottschall, K., \& Voß, G. G. (Hrsg.). (2003). Entgrenzung von Arbeit und Leben. Zum Wandel der Beziehung von Erwerbstätigkeit und Privatsphäre im Alltag. München: Hampp (Arbeit und Leben im Umbruch, 5).

Hanglberger, D. (2010). Arbeitszufriedenheit und flexible Arbeitszeiten. Empirische Analyse mit Daten des Sozio-oekonomischen Panels. Berlin (SOEPpapers, 304): DIW Berlin (Hrsg.).

Jacobs, M. (2016). Reformbedarf im Arbeitszeitrecht. Neue Zeitschrift für Arbeitsrecht, 733-737.

Kauffeld, S. (Hrsg.). (2019). Arbeits-, Organisations- und Personalpsychologie für Bachelor (3. Aufl.). Berlin/Heidelberg: Springer Verlag. 
Kratzer, N., \& Sauer, D. (2003). Entgrenzung von Arbeit. Konzept, Thesen, Befunde. In K. Gottschall \& G. G. Voß (Hrsg.), Entgrenzung von Arbeit und Leben. Zum Wandel der Beziehung von Erwerbstätigkeit und Privatsphäre im Alltag (S. 87-123). München: Hampp (Arbeit und Leben im Umbruch, 5).

Krause, R. (2016). Digitalisierung der Arbeitswelt - Herausforderungen und Regelungsbedarf. Gutachten B zum 71. Deutschen Juristentag. Essen: C. H. Beck (Verhandlungen des 71. Deutschen Juristentages, Band I: Gutachten/Teil B).

Mellner, C. (2016). After-hours availability expectations, work-related smartphone use during leisure, and psychological detachment. International Journal of Workplace Health Management, $9(2), 146-164$.

Lott, Y. (2017). Flexible Arbeitszeiten: Eine Gerechtigkeitsfrage? Hans-Böckler-Stiftung (Hrsg.). Düsseldorf (Forschungsförderung Report, 1).

Richter, M., Kliner, K., \& Rennert, D. (2017). Ergebnisse der BKK-Umfrage „Digitalisierung, Arbeit und Gesundheit“. In F. Knieps \& H. Pfaff (Hrsg.), Digitale Arbeit-digitale Gesundheit. Zahlen, Daten, Fakten (S. 105-124). Berlin: Medizinisch Wissenschaftliche Verlagsgesellschaft.

Rüger, H., \& Ruppenthal, S. (2011). Berufsbedingte räumliche Mobilität - Konsequenzen für Wohlbefinden und Gesundheit. BKK 120-125.

Schicke, A., \& Lauenstein, O. (2016). Flexibel, aber selbstbestimmt - Arbeitszeitwünsche heute. In Bundesministerium für Arbeit und Soziales (BMAS) (Hrsg.), Werkheft 02 - Wie wir arbeiten (wollen) (S. 74-79). Berlin.

Schröder, H. (2019). Digitalisierung - gesundes Arbeiten ermöglichen. Repräsentative Beschäftigtenbefragung. Chartpräsentation zur Pressekonferenz zum Erscheinen des Fehlzeitenreports 2019 am 17. September 2019 in Berlin, Wissenschaftliches Institut der AOK (WIdO).

Schwemmle, M., \& Wedde, P. (2012). Digitale Arbeit in Deutschland: Potenziale und Problemlagen. Bonn: Friedrich-Ebert-Stiftung Medienpolitik.

Schwemmle, M., \& Wedde, P. (2018). Alles unter Kontrolle? Arbeitspolitik und Arbeitsrecht in digitalen Zeiten. In Wiso Diskurs 02/2018. Bonn: Friedrich-Ebert-Stiftung.

Sonnentag, S. (2012). Psychological detachment from work during leisure time. Current Directions in Psychological Science, 21(2), 114-118.

Thüsing, G. (2016). Digitalisierung der Arbeitswelt - Impulse zur rechtlichen Bewältigung der Herausforderung gewandelter Arbeitsformen. Soziales Recht, 3, 87-108.

Voß, G. G. (1998). Die Entgrenzung von Arbeit und Arbeitskraft. Eine subjektorientierte Interpretation des Wandels der Arbeit. Mitteilungen aus der Arbeitsmarkt- und Berufsforschung, 31(3), 473-487.

Zanker, C. (2017). Mobile Arbeit - Anforderungen und tarifliche Gestaltung. Das Beispiel Deutsche Telekom. WSI Mitteilungen, 6/2017, 456-459.

Open Access Dieses Kapitel wird unter der Creative Commons Namensnennung 4.0 International Lizenz (http://creativecommons.org/licenses/by/4.0/deed.de) veröffentlicht, welche die Nutzung, Vervielfältigung, Bearbeitung, Verbreitung und Wiedergabe in jeglichem Medium und Format erlaubt, sofern Sie den/die ursprünglichen Autor(en) und die Quelle ordnungsgemäß nennen, einen Link zur Creative Commons Lizenz beifügen und angeben, ob Änderungen vorgenommen wurden.

Die in diesem Kapitel enthaltenen Bilder und sonstiges Drittmaterial unterliegen ebenfalls der genannten Creative Commons Lizenz, sofern sich aus der Abbildungslegende nichts anderes ergibt. Sofern das betreffende Material nicht unter der genannten Creative Commons Lizenz steht und die betreffende Handlung nicht nach gesetzlichen Vorschriften erlaubt ist, ist für die oben aufgeführten Weiterverwendungen des Materials die Einwilligung des jeweiligen Rechteinhabers einzuholen.

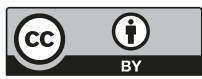

